\title{
Erster AOTrauma-Kurs „Periprothetische Frakturen“ in Berlin
}

\author{
Thomas Fuchs, Michael Schütz
}

\section{Am 2. und 3. März 2017 fand in Berlin in alten Hörsaal der Sportmedizin der Charité der 1. AOTrauma-Kurs „Periprothetische Frakturen“ in Berlin unter der Leitung von Thomas Fuchs und Michael Schütz statt.}

Die beiden Neuen in der Stadt haben mit der Unterstützung von Michael Raschke den Kurs „Periprothetische Frakturen“, der bisher traditionell in Münster stattgefunden hat, erstmalig nach Berlin transferiert. Mehr als 40 Teilnehmer haben an 2 Tagen die aktuellen Herausforderungen periprothetischer Frakturen demonstriert bekommen und aktiv diskutiert. Dabei war das hohe Niveau der Teilnehmer bemerkenswert. Unter den Teilnehmern fanden sich viele Chefärzte, Leitende Oberärzte und Oberärzte. Diese geballte Fachkompetenz zeigte sich vor allem bei den sehr regen, interaktiven Falldiskussionen, die durchweg positiv bewertet wurden.

Die Vorträge, Falldiskussionen in Kleingruppen sowie die praktischen Übungen wurden durch eine hochmotivierte Faculty begleitet. Unter anderem 7 Ordinarien waren gekommen, um ihre Strategien zur Lösung des Problems „periprothetische Fraktur“ zu präsentieren. Dabei gelang es, eine kommunikative Atmosphäre zu schaffen, in der die vorgestellten Vorgehensweisen auch kritisch hinterfragt wurden.

Am 1. Kurstag wurde durch den Präsidenten der AOTrauma Deutschland, Prof. Michael Raschke, ein Faktencheckvortrag über periprothetische Frakturen gehalten, der noch einmal verdeutlichte, welch enorme Herausforderung vor uns liegt und dass noch lange nicht alle Probleme gelöst sind.

In fast allen Beiträgen stand fast immer die gleiche Frage im Raum, die die Behandlungsstrategie bei Vorliegen einer periprothetischen Fraktur bestimmt: Ist die Prothese fest oder locker? Und wenn sie locker ist, war sie es schon vor dem Trauma? Könnte eine Infektion Ursache für eine Lockerung sein? Wie bekommen wir heraus, ob eine Infektion vorliegt? Thomas Mittlmeier (Rostock), UIrich Stöckle (Tübingen) und Andrej Trampuz (Berlin) widmeten sich bei ihren Beträgen dieser Herausforderung.

Parallel zu den praktischen Übungen, in denen Tipps und Tricks bei der operativen Versorgung vermittelt wurden, lockten Wolfgang Ertel (Berlin), Klaus-Dieter Schaser (Dresden) und Reinhold Laun (Berlin) die Teilnehmer mit ausgewählten Fällen aus ihrem klinischen Alltag aus der Reserve. Dabei wurde deutlich, dass auch einmal unkon-

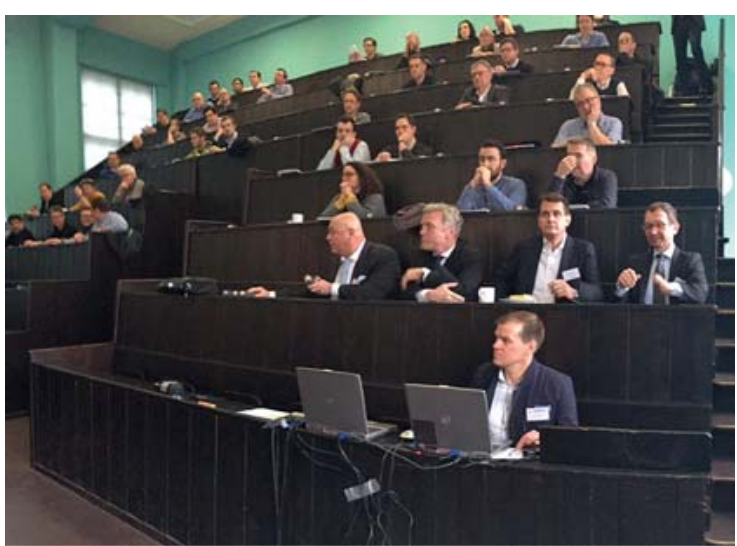

- Abb. 1 Alter Hörsaal der Sportmedizin. Bild: AOTrauma Deutschland.

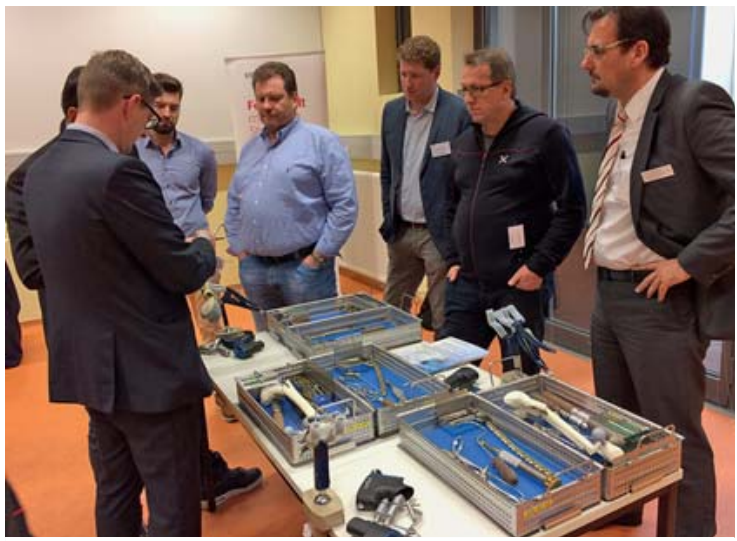

Abb. 2 Praktische Übungen. Bild: AOTrauma Deutschland. 


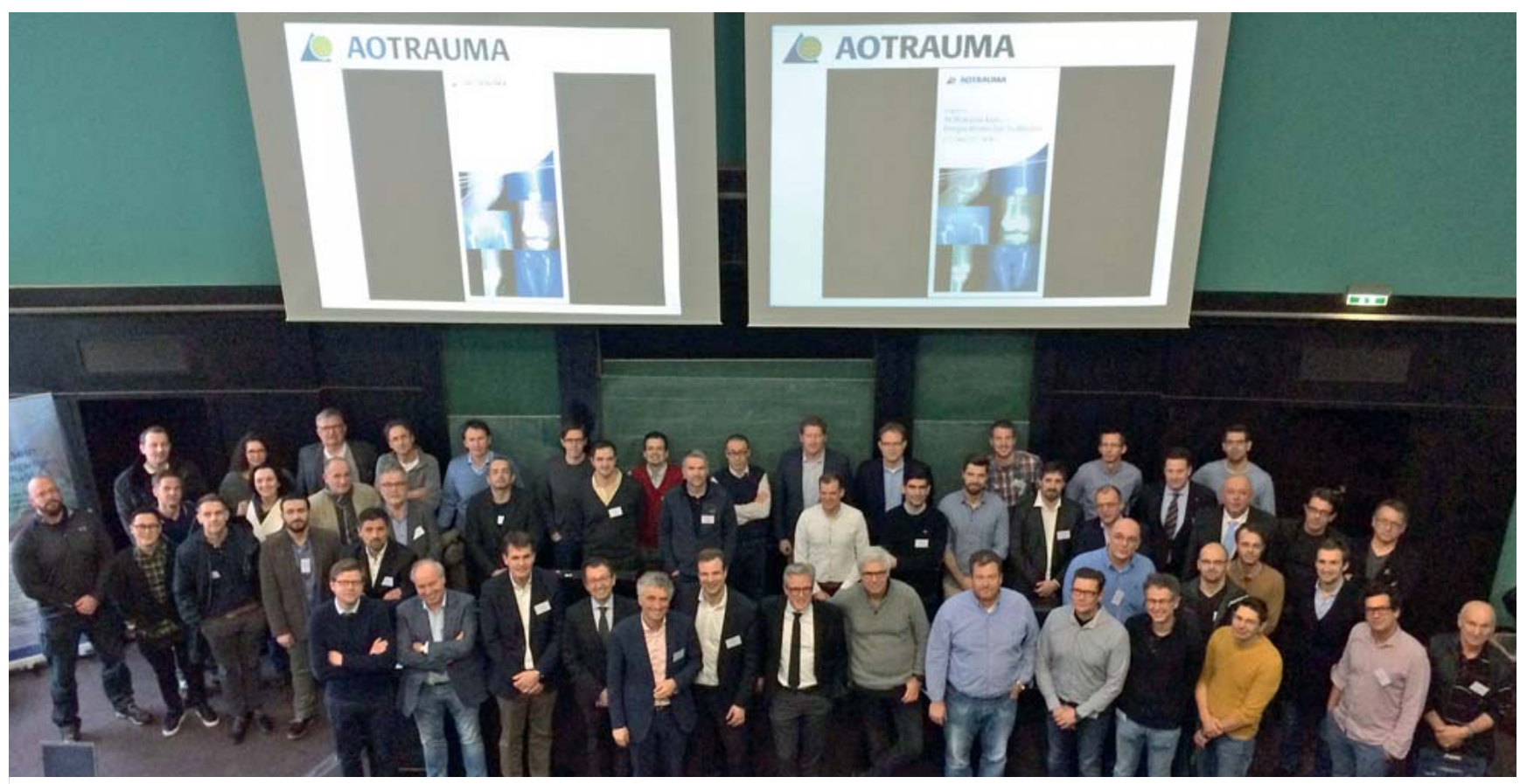

Abb. 3 Faculty und Teilnehmer. Bild: AOTrauma Deutschland.

ventionelle Wege beschritten werden müssen, um bei der Behandlung erfolgreich zu sein.

Abends haben dann Faculty und Teilnehmer gemeinsam ein Stück Italien in der Mitte von Berlin erleben können. In alter AO-Tradition wurden neben fachlichen Dingen auch persönliche Kontakte geknüpft und gefestigt.

Der 2. Tag begann mit den selteneren periprothetischen Frakturen. Allerdings stellte sich nach dem Vortrag von Markus Scheibel heraus, dass periprothetische Frakturen der Schulter gar nicht so selten sind und ihre Zahl über die letzten Jahre rasant ansteigt.

Georg Matziolis (Eisenberg) und Stefan Tohtz (Berlin) haben in der 2. Sitzung des Tages die Besonderheiten der Revisionsendoprothetik an Knie und Hüfte dargestellt, und auch hier wurde in der anschließenden Diskussion deutlich, wie komplex mitunter die Probleme sind und dass es nicht immer die richtige Antwort gibt.

Den abschließenden Vortrag hielt Klaus-Dieter Schaser (Dresden). In seinem Beitrag wurde die Frage von Nachbehandlungsstandards bei periprothetischen Frakturen behandelt. Bemerkenswert war die Erkenntnis, welche
Kräfte schon bei Lagerungsmaßnahmen und Transfer mit vermeintlicher Entlastung auf Osteosynthesen wirken.

Die Resonanz der Teilnehmer war durchweg positiv, sodass als Fazit ein gelungener 1. AOTrauma-Kurs „Periprothetische Frakturen“ in Berlin bleibt. Im Jahr 2018 wird der Kurs wieder in Berlin stattfinden.

Korrespondenzadresse

\section{PD Dr. Thomas Fuchs}

Vivantes Klinikum im Friedrichshain

Berlin

thomas.fuchs@vivantes.de

Univ.-Prof. Dr. Michael Schütz

Universitätsklinikum Charité

Berlin

michael.schuetz@charite.de

Bibliografie

DOI https://doi.org/10.1055/s-0043-109138

OP-JOURNAL 2017; 33: 82-83 @ Georg Thieme Verlag KG Stuttgart · New York ISSN 0178-1715 